\title{
A mysterious tumor in the obturator internus muscle - a case report
}

Marcin Pachowicz¹, Anna Drelich-Zbroja², Justyna Szumiło³, Szymon Skwarcz ${ }^{4}$, Beata Chrapko ${ }^{1}$

${ }^{1}$ Nuclear Medicine Department, Medical University of Lublin, Poland

${ }^{2}$ Neuroradiology and Invasive Radiology Department, Medical University of Lublin, Poland

${ }^{3}$ Clinical Pathomorphology Department, Medical University of Lublin, Poland

${ }^{4}$ Orthopedics and Traumatology Department, Medical University of Lublin, Poland

[Received 4 VI 2016; Accepted 9 I 2017]

\section{Case report}

A 33-year-old male was admitted to local Nuclear Medicine Department with suspected femoral head necrosis. His chief complaint was pain located in left inguinal region exacerbating during walking. He was forced to use a crutch.

Three-phase bone scan (TPBS) was performed according to the standard protocol used in our Department. ${ }^{99 m}$ Tc-labelled

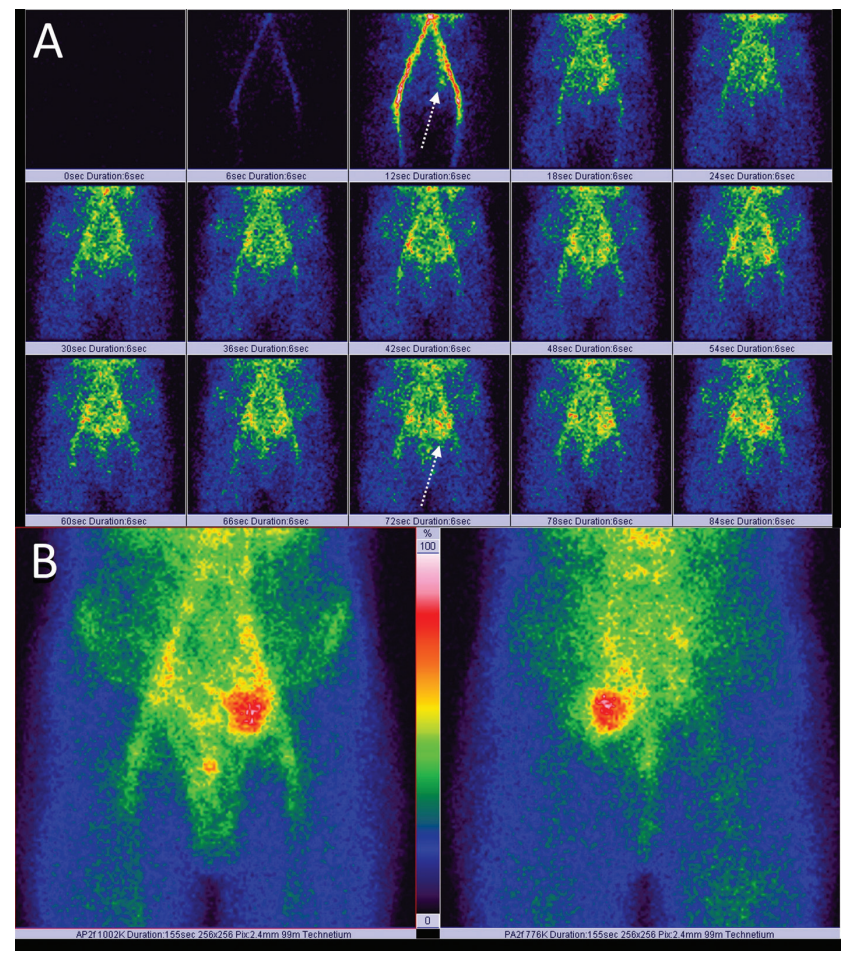

Figure 1. A. TPBS — blood flow images. AP view. Note hyperperfusion in area medially to the left iliac arteries (arrow); B. TPBS - blood pool images. AP and PA views. Area of significant hyperemia medially to the left hip joint

\section{Correspondence to: Marcin Pachowicz}

Nuclear Medicine Department, Medical University of Lublin

ul. K. Jaczewskiego 8c (SPSK NR 4), 20-090 Lublin, Poland

Tel: +48817244339

Fax: +48817244339

E-mail: marcin.pachowicz.md@gmail.com methylene diphosphonate (MDP) was used, administered activity was $740 \mathrm{MBq}$. The TBPS was followed by SPECT/CT scan of the pelvis. The results are presented in Figures 1 and 2 .

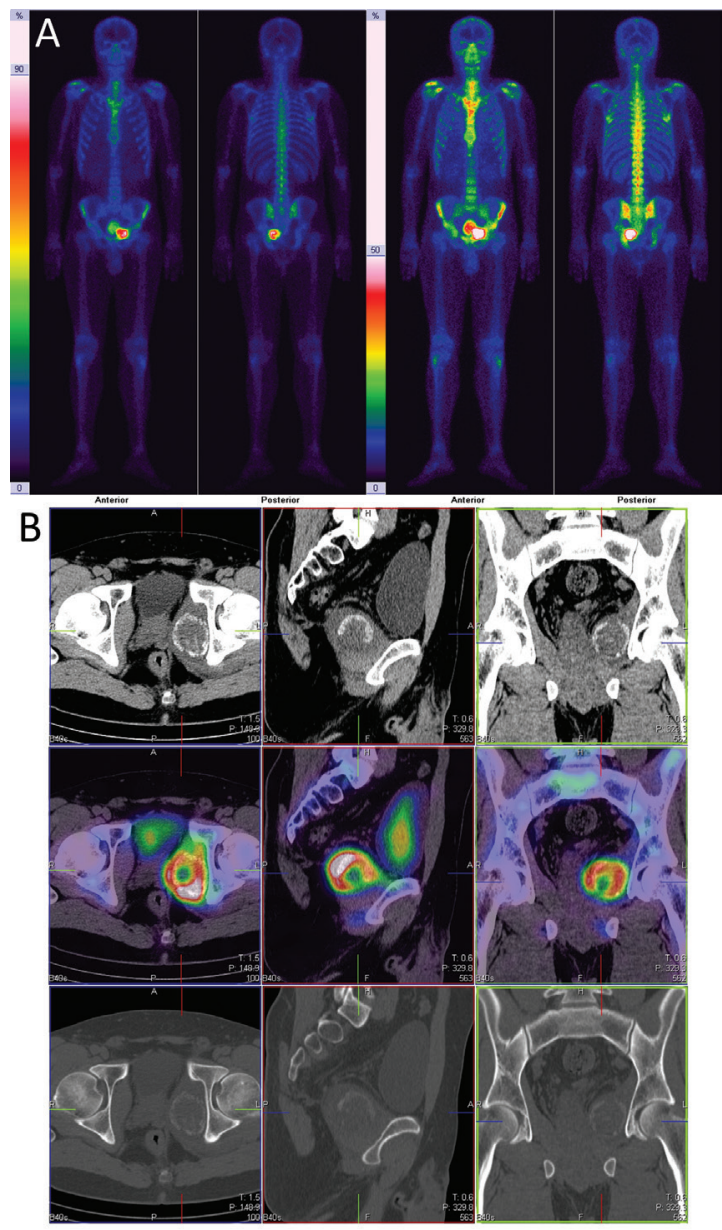

Figure 2. A. Whole body bone scan (dual intensity projection). High MDP accumulation in the area between bladder and left hip joint. Increased radiotracer accumulation in the adjacent bone (ischium). Slightly and irregularly increased MDP uptake in the distal shaft of the right humerus was also observed - probably due to use of a crutch; B. SPECT/CT images clearly indicate radiotracer accumulation in the tumor located within the left obturator internus muscle. Slightly increased accumulation in the adjacent bones with no visible destruction of bone structure. The tumor itself is polycyclic in shape and highly calcified, compresses surrounding structures, but without signs of infiltration 
Caution and further evaluation recommended as malignancy were suspected, so MRI scan was executed. The results are presented in Figure 3 .

Basing on clinical appearance and obtained images the decision of surgery was made. The surgeon removed a brown

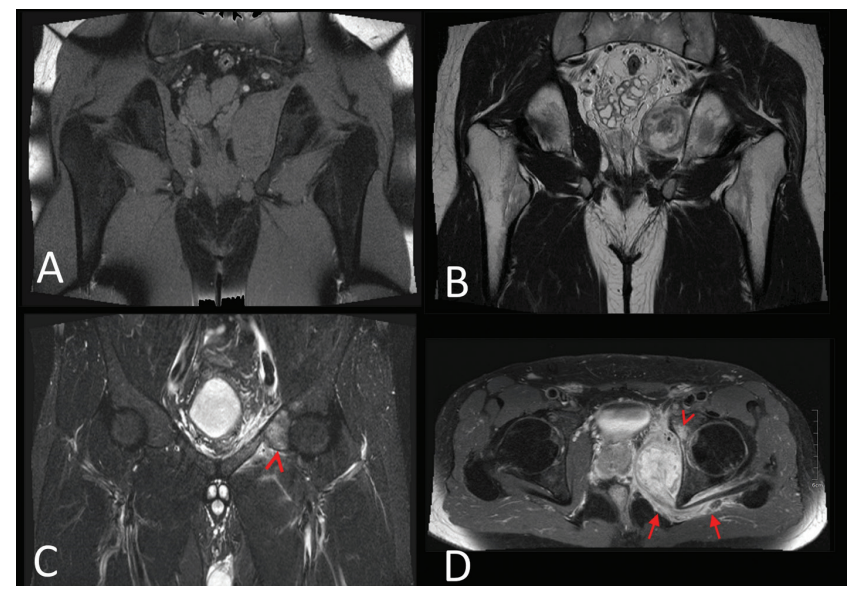

Figure 3. A. MRI T1-weighted TSE-FATSAT images, coronal section. The abnormal size of the obturator internus muscle associated with compression of the prostate, urinary bladder and seminal vesicles. The muscle itself has rather homogenous, isointensive signal; $\mathbf{B}$. MRI T2-weighted TSE images, coronal section. Nonhomogenous hyperintensive tumor filling nearly entire obturator internus muscle. No evidence of infiltration. Adjacent bone intact; C. MRI T2-weighted TSE TIRM images, coronal section. Hyperintense area medially to the acetabulum (arrowhead) - edema; D. MRI T1- weighted TSE TRA+FATSAT $+C$ images. Axial section. Increased signal from the entire obturator internus muscle indicating edema (arrows). Hyperintense area within the acetabulum and pubis also indicate edema (arrowhead) tumor. Primary histopathological verification stated: mesenchymal chondrosarcoma, however, further and more detailed evaluation, including immunohistochemical reactions, suggested fibroblastic osteosarcoma rather than chondrosarcoma.

Six months after the surgery and chemotherapy the patient underwent ${ }^{18} \mathrm{~F}$-FDG-PET/CT scan to rule out local recurrence or distant metastases. The results are presented in Figure 4.

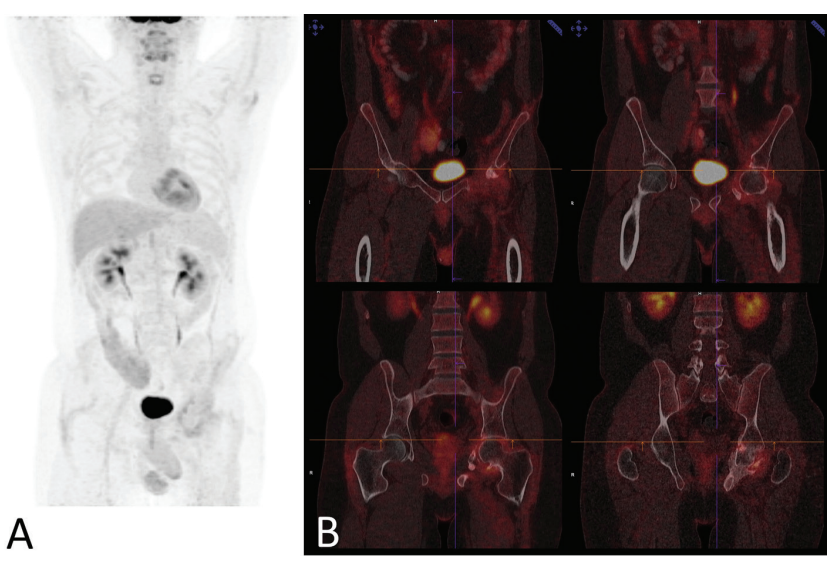

Figure 4. A. MIP PET scan after surgery and chemotherapy. Physiological glucose uptake and excretion by kidneys. Increased tonsils, laryngeal muscles and bowel uptake - normal variant. Slightly increased and irregular glucose uptake in the operation area consistent with post-operative changes. No distant metastases; B. PET/CT scans. Coronal sections. No pathologic, glucose avid mass suggesting local recurrence within pelvis. Post-operative changes. Physiologic activity in the urinary bladder (excreted radioactive glucose) 